NISSUNA UMANA INVESTIGAZIONE SI PUO DIMANDARE VERA SCIENZIA S'ESSA NON PASSA PER LE MATEMATICHE DIMOSTRAZIONI LEONARDO DA VINCI

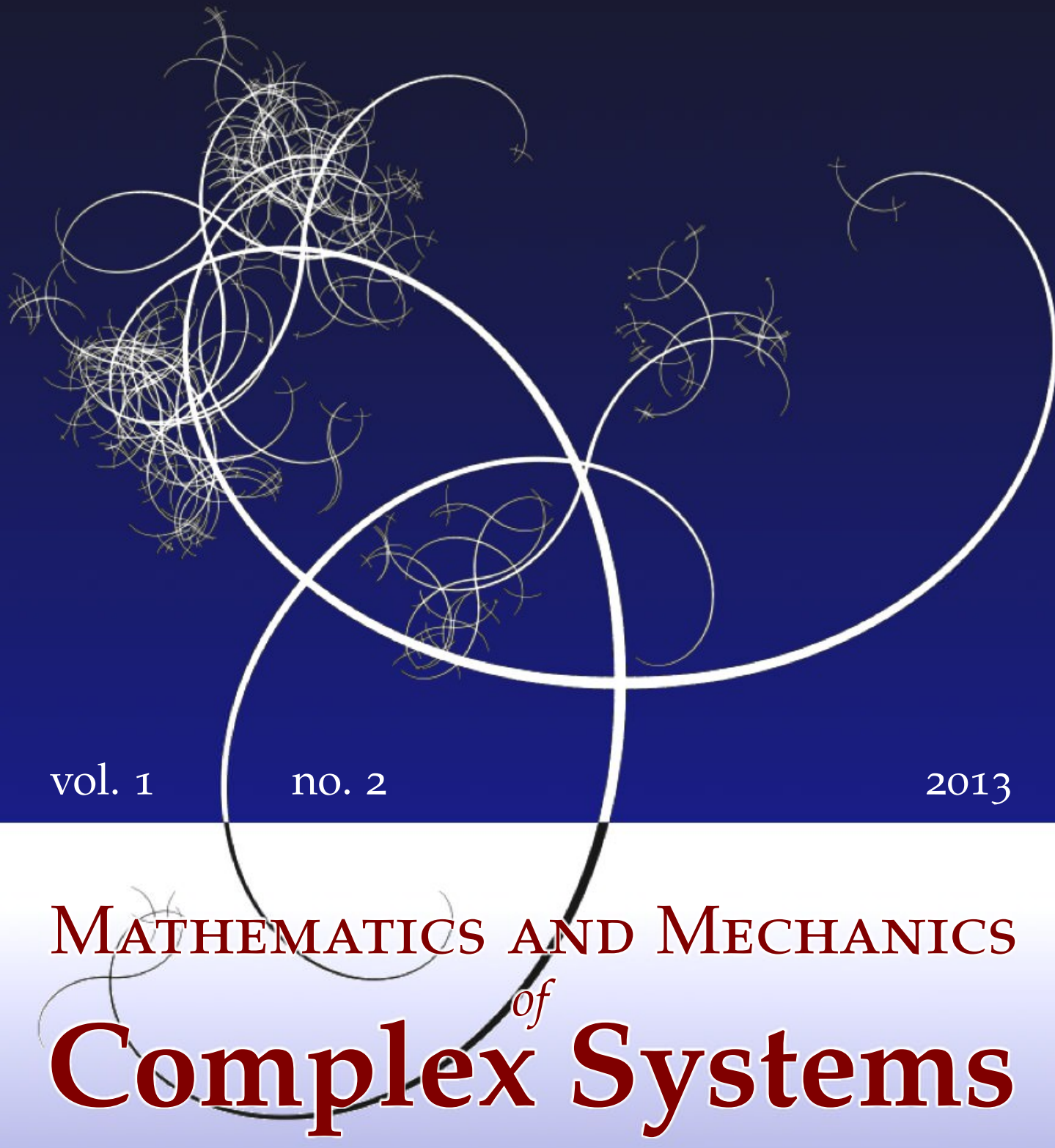

Fedor L. Bakharev, Sergey A. NaZarov and Guido H. Sweers A SUFFICIENT CONDITION FOR A DISCRETE SPECTRUM OF THE KIRCHHOFF PLATE WITH AN INFINITE PEAK 


\title{
A SUFFICIENT CONDITION FOR A DISCRETE SPECTRUM OF THE KIRCHHOFF PLATE WITH AN INFINITE PEAK
}

\author{
Fedor L. Bakharev, Sergey A. Nazarov and Guido H. Sweers
}

Sufficient conditions for a discrete spectrum of the biharmonic equation in a twodimensional peak-shaped domain are established. Different boundary conditions from Kirchhoff's plate theory are imposed on the boundary and the results depend both on the type of boundary conditions and the sharpness exponent of the peak.

\section{Motivation}

Elliptic boundary value problems on domains which have a Lipschitz boundary and a compact closure, in particular when they generate positive self-adjoint operators, have fully discrete spectra. However, if the domain loses the Lipschitz property or compactness, other situations may occur. It is well-known that for the Dirichlet case boundedness is sufficient but not necessary for having discrete spectrum. See the famous paper [Rellich 1948] or the more recent [Rozenbljum 1972; van den Berg 1984]. On the other hand, for the Neumann problem of the Laplace operator there exist numerous examples of bounded domains such that the spectrum gets a nonempty continuous component (see e.g. [Courant and Hilbert 1953; Maz'ya and Poborchii 2006; 1997; Simon 1992; Hempel et al. 1991]).

The literature on the spectra for the Laplace operator with various boundary conditions on special domains is focused on domains that have a cusp, a finite or infinite peak or horn [van den Berg 1984; Hempel et al. 1991; Jakšić et al. 1992; Davies and Simon 1992; Jakšić 1993; Ivrii 1999; Boyarchenko and Levendorskii 2000; van den Berg and Lianantonakis 2001; Kovař́k 2011] or even a rolled horn [Simon 1992].

The criteria in [Adams and Fournier 2003] and [Evans and Harris 1987] for the embedding $H^{1}(\Omega) \subset L_{2}(\Omega)$ to be compact show that the Neumann-Laplace

This work is supported by the Russian Foundation for Basic Research (project 12-01-00348). Bakharev is also supported by the Chebyshev Laboratory (Department of Mathematics and Mechanics, Saint Petersburg State University) under the grant 11.G34.31.0026 of the Government of the Russian Federation and grant 6.38.64.2012 of Saint Petersburg State University.

MSC2010: 35P05, 47A10, 74K20.

Keywords: Kirchhoff plate, cusp, peak, discrete and continuous spectra. 
problem on a domain $\Omega$ with the infinite peak

$$
\Pi_{R}=\left\{x=\left(x_{1}, x_{2}\right) \in \mathbb{R}^{2}: x_{1}>R,-H\left(x_{1}\right)<x_{2}<H\left(x_{1}\right)\right\},
$$

where the function $H>0$ is smooth and monotone decreasing, has discrete spectrum if and only if

$$
\lim _{y \rightarrow+\infty} \int_{y}^{+\infty} \frac{H(\eta)}{H(y)} d \eta=0 \quad\left(\Longleftrightarrow \lim _{y \rightarrow+\infty} \frac{H(y+\epsilon)}{H(y)}=0 \text { for any } \epsilon>0\right) .
$$

The function $H$ is assumed to have a first derivative that tends to zero and a bounded second derivative. It will be convenient to use the notation $\Upsilon(y)=(-H(y), H(y))$. Here is an image of such a domain:

The simplest boundary irregularity violating the Lipschitz condition is just the (finite) peak

$$
\varpi_{R}=\left\{x: 0<x_{1}<R,-h\left(x_{1}\right)<x_{2}<h\left(x_{1}\right)\right\},
$$

where $h\left(x_{1}\right)=h_{0} x_{1}^{1+\alpha}, h_{0}>0$ and $\alpha>0$. Nevertheless, the spectrum of the Neumann problem in the domain with this peak stays discrete. See Remark 5.1.

A criterion ([Nazarov 2009]) for having essential spectrum in the Neumann problem for elliptic systems of second order differential equations with a polynomial property is derived in [Nazarov 1999]. In particular it shows that the continuous spectrum of an elastic body with $\alpha \geq 1$ for the peak (3) is nonempty (see [Nazarov 2008; Bakharev and Nazarov 2009]). This phenomenon of generating wave processes in a finite volume, is known experimentally and used in the engineering practice to construct wave dampers, "black holes", for elastic oscillations (see [Mironov 1988; Krylov and Tilman 2004], etc.).

In this paper we study the spectra of boundary value problems for the Kirchhoff model of a thin elastic plate described by the biharmonic operator $\Delta^{2}$. The boundary conditions that we consider model the three mechanically most reasonable cases, namely where the lateral sides of the peak are supplied with one of the following three types of the boundary conditions: clamped edge (Dirichlet), traction-free edge (Neumann) and hinged edge (Mixed). In all these cases the spectrum of the problem in a bounded domain with the peak as in (3) is discrete. We derive sufficient conditions for the spectrum to be discrete for the boundary value problem on an unbounded domain with a peak as in (1).

If a sufficient number of Dirichlet conditions are imposed on the lateral sides of the peak (the cases $\boldsymbol{D}-\boldsymbol{N}, \boldsymbol{M}-\boldsymbol{M}, \boldsymbol{D}-\boldsymbol{M}$, and $\boldsymbol{D}-\boldsymbol{D}$; see formulas (5)-(7) and 
(11), (12)), then the proof that the spectrum is discrete becomes rather simple (Theorem 5). Indeed, it suffices to apply the weighted Friedrich's inequality (13) and to take into account the decay of the quantity $H(y)$ as $y \rightarrow+\infty$.

Our main interest concerns the cases $\boldsymbol{M}-\boldsymbol{N}$ and $\boldsymbol{N}-\boldsymbol{N}$. By applying weighted inequalities of Hardy type (Lemmas 7 and 8) we obtain a sufficient condition for the case $\mathbf{N}-\boldsymbol{N}$ to have discrete spectrum (Proposition 4). Indeed, as shown in [Adams and Fournier 2003], the second condition in (2) implies a criterion for the compact embedding $H^{m}(\Omega) \subset L_{2}(\Omega)$ for all $m$ (we just need $m=2$ ). One can also use that approach (Theorem 12) for the case $\boldsymbol{M}-\boldsymbol{N}$. This approach differs from the one used in [Adams and Fournier 2003; Evans and Harris 1987]. The different argument allows to obtain a condition for having discrete spectrum if one of the peak's edges is traction-free and the other one is hinged (the case $\boldsymbol{N}-\boldsymbol{M}$; see Theorem 13).

The obtained results essentially differ from each other: under the conditions (11) and also under (12) any decay of $H$ is enough, the case $\boldsymbol{M}-\boldsymbol{N}$ needs a power decay rate with the exponent $\alpha>1$, while the case $N-N$ needs a superexponential decay rate. See Remark 12.1 and 13.1.

\section{The Kirchhoff plate model}

Assumption 1. Let $\Omega$ be a domain in the plane $\mathbb{R}^{2}$ with a smooth (of class $C^{\infty}$ ) boundary $\Gamma$ such that, for some $R>0$ and some monotone decreasing function $H:[R, \infty) \rightarrow \mathbb{R}^{+}$with $\lim _{t \rightarrow \infty} H(t)=0$,

(1) $\left\{\left(x_{1}, x_{2}\right) \in \Omega ; x_{1}>R\right\}=\left\{\left(x_{1}, x_{2}\right) ; x_{1}>R\right.$ and $\left.\left|x_{2}\right|<H\left(x_{1}\right)\right\}$ and

(2) $\left\{\left(x_{1}, x_{2}\right) \in \Omega ; x_{1}<R\right\}$ is bounded.

We regard $\Omega$ as the projection of a thin isotropic homogeneous plate and apply the Kirchhoff theory (see [Mikhlin 1970, §30], [Nazarov 2002, Chapter 7], and so on). So we arrive at the fourth-order differential equation

$$
\Delta^{2} u(x)=\lambda u(x), \quad x \in \Omega,
$$

which describes transverse oscillations of the plate. Here, $u(x)$ is the plate deflection, and $\lambda$ a spectral parameter proportional to the square of the oscillation frequency.

The following sets of boundary conditions have a clear physical interpretation (see [Mikhlin 1970, §30], [Gazzola et al. 2010, §1.1], and so on):

(D): Dirichlet for a clamped edge:

$$
u(x)=\partial_{n} u(x)=0, \quad x \in \Gamma_{D} .
$$


$(N)$ : Neumann for a traction-free edge:

$$
\left\{\begin{array}{c}
\partial_{n} \Delta u(x)-(1-v)\left(\partial_{s} \varkappa(x) \partial_{s} u(x)-\partial_{s}^{2} \partial_{n} u(x)\right)=0, \\
\Delta u(x)-(1-v)\left(\partial_{s}^{2} u(x)+\varkappa(x) \partial_{n} u(x)\right)=0,
\end{array} \quad x \in \Gamma_{N} .\right.
$$

$(M)$ : Mixed for a hinged edge:

$$
u=\Delta u(x)-(1-v) \varkappa(x) \partial_{n} u(x)=0, \quad x \in \Gamma_{M} .
$$

Here, $\partial_{n}$ and $\partial_{s}$ stand for the normal and tangential derivatives, $\varkappa(x)$ is the signed curvature of the contour $\Gamma$ at the point $x \in \Gamma$ positive for convex boundary parts, and $v \in[0,1 / 2)$ is the Poisson ratio. Finally, $\Gamma_{D}, \Gamma_{N}$, and $\Gamma_{M}$ are the unions of finite families of open curves and $\Gamma=\overline{\Gamma_{D}} \cup \overline{\Gamma_{N}} \cup \overline{\Gamma_{M}}$, two of which may be empty. In what follows it is convenient to use the notation $y=x_{1}$ and $z=x_{2}$.

The general properties of the spectra depend on which of the boundary conditions (5)-(7) are imposed on the upper (+) and lower (-) sides,

$$
\Sigma_{R}^{ \pm}=\{x: y>R, z= \pm H(y)\}
$$

of the peak. Let us give a precise statement. We define a symmetric bilinear form on $H^{2}(\Omega)$ by

$$
a(u, u)=\int_{\Omega}\left(\left|\frac{\partial^{2} u}{\partial x_{1}^{2}}\right|^{2}+\left|\frac{\partial^{2} u}{i} \partial x_{2}^{2}\right|^{2}+2(1-v)\left|\frac{\partial^{2} u}{\partial x_{1} \partial x_{2}}\right|^{2}+2 v \frac{\partial^{2} u}{\partial x_{1}^{2}} \frac{\partial^{2} u}{\partial x_{2}^{2}}\right) d x
$$

and $a(u, v)=\frac{1}{4} a(u+v, u+v)-\frac{1}{4} a(u-v, u-v)$. Then $\frac{1}{2} a(u, u)$ is the elastic energy stored in the plate. Since one directly verifies that

$$
a(u, u) \geq(1-v) \sum_{j, k=1}^{2} \int_{\Omega}\left|\frac{\partial^{2} u}{\partial x_{j} \partial x_{k}}\right|^{2} d x,
$$

the bilinear form $a(\cdot, \cdot)$ is nonnegative.

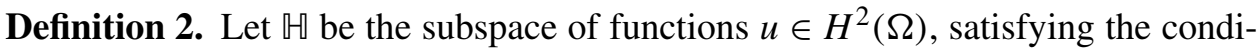
tions (5) on $\Gamma_{D}$ and $u=0$ on $\Gamma_{M}$ in the sense of traces.

By [Birman and Solomyak 1980, §10.1] $\mathbb{W}$ is a Hilbert space with scalar product $a(\cdot, \cdot)+(\cdot, \cdot)_{\Omega}$, where $(\cdot, \cdot)_{\Omega}$ is the standard scalar product in the Lebesgue space $L_{2}(\Omega)$. Moreover, Theorem 2 of [Birman and Solomyak 1980, §10.1] implies that there exists a unique (unbounded) self-adjoint operator $A: D(A) \subset$ $L_{2}(\Omega) \rightarrow L_{2}(\Omega)$ with $D(A) \subset D\left(A^{1 / 2}\right)=\mathbb{H}$ such that

$$
(A u, v)_{\Omega}=a(u, v) \text { for all } v \in \mathbb{H} .
$$


Note that the eventually remaining boundary conditions in (5)-(7) appear in $D(A) \subset$ $H^{4}(\Omega)$ as intrinsic natural boundary conditions from (9)-(8), see again [Mikhlin 1970, §30], [Gazzola et al. 2010, §1.1] etc.

Definition 3. By the spectrum for (4)-(7) we will mean $\sigma(A)$ with $A$ defined in (10).

Since $a$ is nonnegative, the spectrum $\sigma(A)$ belongs to $[0, \infty)$.

As a direct consequence of known results we may state the following.

Proposition 4. If (2) holds, then the spectrum of the Equation (4), with either of the above boundary condition on the sides of the peak, is discrete.

Proof. By [Birman and Solomyak 1980, $\$ 10.1$ Theorem 5] the spectrum is discrete if and only if the embedding $\mathbb{\hookrightarrow} \hookrightarrow L_{2}(\Omega)$ is compact. By [Adams and Fournier 2003; Evans and Harris 1987] one knows that $H^{2}(\Omega) \hookrightarrow L_{2}(\Omega)$ is compact whenever (2) holds true and $\mathbb{H} \subset H^{2}(\Omega)$.

\section{Simple cases: $D-N, M-M, D-M$ and $D-D$,}

Theorem 5. Suppose $\Omega$ is as in Assumption 1 and suppose that the boundary conditions for problem (4), as given in (5)-(7) contain one of the cases (11) or (12). Then the spectrum is discrete.

Remark 5.1. By a similar reasoning, we may conclude that in the bounded domain $\omega$, with the peak as in (3), Equation (4) has discrete spectrum for any set of conditions (5)-(7) on the arc $\partial \omega \backslash \mathcal{O}$. This fact follows from the inequality (see [Nazarov and Taskinen 2008]):

$$
\left\||x|^{-1} u ; L_{2}(\omega)\right\|^{2} \leq c\left(\left\|\nabla u ; L_{2}(\omega)\right\|^{2}+\left\|u ; L_{2}\left(\omega \backslash \varpi_{R}\right)\right\|^{2}\right) .
$$

Proof. By assumption the boundary conditions provide at least one of the following two groups of relations:

$$
\begin{array}{ll}
u=0 & \text { on } \Sigma_{R}^{+} \cup \Sigma_{R}^{-} ; \\
u=\partial_{n} u=0 & \text { on } \Sigma_{R}^{+} \text {or on } \Sigma_{R}^{-} .
\end{array}
$$

In both cases (11) and (12) the following version of Friedrich's inequality is valid:

$$
\int_{\Upsilon(y)}\left|\frac{\partial^{2} u}{\partial z^{2}}(y, z)\right|^{2} d z \geq \frac{c}{H(y)^{4}} \int_{\Upsilon(y)}|u(y, z)|^{2} d z .
$$

Therefore,

$$
a(u, u) \geq c \int_{\Omega} H(y)^{-4}|u(x)|^{2} d x .
$$


The embedding operator $\gamma: \mathbb{H} \rightarrow L_{2}(\Omega)$ can be represented as the sum $\gamma_{0}+\gamma_{\rho}$, where $\rho \geq R$ is large and positive, $\gamma_{0}=\gamma-\gamma_{\rho}$, and $\gamma_{\rho}$ contains the operator of multiplication by the characteristic function of $\Pi_{\rho}$. The operator $\gamma_{0}$ is compact, and the norm of $\gamma_{\rho}$, in view of (14), does not exceed $c \max \left\{H\left(x_{1}\right)^{-2} ; x_{1} \geq \rho\right\}$. Since the function $H$ decays, this quantity goes to zero when $\rho \rightarrow+\infty$, i.e., the operator $\gamma$ can be approximated by compact operators in the operator norm. Thus $\gamma$ is compact and the result is proved.

\section{Auxiliary inequalities}

First of all we prove some one-dimensional weighted inequalities, two of which are of Hardy type involving a weight function $h$ as follows.

Assumption 6. Let h be a positive weight function of class $C^{2}$ on $[0,+\infty)$ such that

$\cdot \int_{0}^{\infty} h(s) d s<\infty$ and

- for some large $T$, we have $h^{\prime}(t)<0$ and $h^{\prime \prime}(t)>0$ for $t \in(T, \infty)$.

Throughout this section $h$ is supposed to satisfy this assumption.

Lemma 7. If $U$ is differentiable for $y \geq R$ and $U(R)=0$, then

$$
\int_{R}^{+\infty} h(y)|U(y)|^{2} d y \leq \int_{R}^{+\infty} F_{h}(y)\left|\partial_{y} U(y)\right|^{2} d y
$$

where

$$
F_{h}(y)=\frac{4}{h(y)}\left(\int_{y}^{+\infty} h(\tau) d \tau\right)^{2} .
$$

Proof. Using the Cauchy-Bunyakovsky-Schwarz inequality, we have

$$
\begin{aligned}
& \int_{R}^{+\infty} h(y)|U(y)|^{2} d y \\
& \quad=2 \int_{R}^{+\infty} h(y) \int_{R}^{y} \partial_{t} U(t) U(t) d t d y \\
& \quad \leq 2 \int_{R}^{+\infty} \int_{t}^{+\infty} h(y)\left|\partial_{t} U(t) U(t)\right| d y d t \\
& \quad \leq 2\left(\int_{R}^{+\infty} h(t)|U(t)|^{2} d t\right)^{1 / 2}\left(\int_{R}^{+\infty} h(t)^{-1}\left(\int_{t}^{+\infty} h(y) d y\right)^{2}\left|\partial_{t} U(t)\right|^{2} d t\right)^{1 / 2},
\end{aligned}
$$

and the result follows through division by a common factor. 
Lemma 8. If $U$ is differentiable for $y \geq R$ and $U(R)=0$, then

$$
\int_{R}^{+\infty} h(y)\left|\partial_{y} U(y)\right|^{2} d y \geq \int_{R}^{+\infty} G_{h}(y)|U(y)|^{2} d y
$$

where

$$
G_{h, R}(y)=\frac{1}{4 h(y)}\left(\int_{R}^{y} h(\tau)^{-1} d \tau\right)^{-2} .
$$

Proof. For functions $v$ with $v(0)=0$ the Hardy inequality tells us that

$$
\int_{0}^{+\infty} t^{-2}|v(t)|^{2} d t \leq 4 \int_{0}^{+\infty}\left|\partial_{t} v(t)\right|^{2} d t
$$

We make the change $t \mapsto y \in[R,+\infty)$ where $t=\int_{R}^{y} h(\tau)^{-1} d \tau$, and set $U(y)=$ $v(t)$. Then $\partial_{t} v(t)=h(y) \partial_{y} U(y)$ leads to the desired estimate.

Corollary 9. If the function $U$ is twice differentiable for $y \geq R$ and $U(R)=$ $U^{\prime}(R)=0$, then

$$
\int_{R}^{+\infty} h(t)\left|\partial_{t}^{2} U(t)\right|^{2} d t \geq W_{h}(R) \int_{R}^{+\infty} h(t)|U(t)|^{2} d t
$$

is valid with

$$
\begin{aligned}
W_{h}(R) & :=\inf _{t \in[R,+\infty)} \frac{G_{h, R}(t)}{F_{h}(t)} \\
& =\inf _{t \in[R,+\infty)} \frac{1}{16}\left(\int_{R}^{t} h(\tau)^{-1} d \tau\right)^{-2}\left(\int_{t}^{\infty} h(\tau) d \tau\right)^{-2} .
\end{aligned}
$$

Lemma 10. We have

$$
\inf _{t \in[R,+\infty)} \frac{G_{h^{3}, R}(t)}{h(t) h^{\prime}(t)^{2}} \geq 1
$$

Suppose moreover that

$$
\lim _{t \rightarrow+\infty} \partial_{t}(\log h(t))=-\infty
$$

Then

$$
W_{h}(R) \rightarrow+\infty \quad \text { and } \quad W_{h^{3}}(R) \rightarrow+\infty \quad \text { for } R \rightarrow \infty .
$$

Proof. Since $-h^{\prime}(\tau) \geq-h^{\prime}(t)>0$ for $\tau<t$, we find

$$
\begin{aligned}
\frac{G_{h^{3}, R}(t)}{h(t) h^{\prime}(t)^{2}} & =\frac{1}{4 h^{\prime}(t)^{2} h(t)^{4}}\left(\int_{R}^{t} h(\tau)^{-3} d \tau\right)^{-2} \geq \frac{1}{4 h(t)^{4}}\left(-\int_{R}^{t} h^{\prime}(\tau) h(\tau)^{-3} d \tau\right)^{-2} \\
& =\frac{1}{4 h(t)^{4}}\left(\frac{1}{2 h(t)^{2}}-\frac{1}{2 h(R)^{2}}\right)^{-2} \geq 1
\end{aligned}
$$


Since (18) equals $h^{\prime}(t) / h(t) \rightarrow-\infty$ for $t \rightarrow \infty$, we find that for $t \rightarrow \infty$ both

$$
h(t)\left(\int_{t}^{\infty} h(\tau) d \tau\right)^{-1} \rightarrow \infty \quad \text { and } \quad \frac{1}{h(t)}\left(\int_{R}^{t} \frac{1}{h(\tau)} d \tau\right)^{-1} \rightarrow \infty .
$$

Hence

$$
\frac{G_{h, R}(t)}{F_{h}(t)} \rightarrow \infty \quad \text { as } t \rightarrow \infty,
$$

and since the quotient also goes to infinity for $t \downarrow R$, it has a minimum in some $t_{R} \in(R, \infty)$. Calculating $\left(G_{h, R}(t) / F_{h}(t)\right)^{\prime}=0$ we find

$$
\frac{1}{h(t)} \int_{t}^{\infty} h(\tau) d \tau-h(t) \int_{R}^{t} h(\tau)^{-1} d \tau=0 .
$$

Hence

$$
\inf _{t \in[R, \infty)}\left(\int_{R}^{t} h(\tau)^{-1} d \tau \int_{t}^{\infty} h(\tau) d \tau\right)^{-1}=h\left(t_{R}\right)^{2}\left(\int_{t_{R}}^{\infty} h(\tau) d \tau\right)^{-2},
$$

which goes to infinity for $R \rightarrow \infty$ since $t_{R}>R$. The claim for $W_{h}(R)$ follows. The same argument holds true for $W_{h^{3}}(R)$.

\section{Estimates for a traction-free boundary}

We assume that the Neumann boundary conditions (6) are imposed at the both sides of the peak (1). Let us describe for $\rho \rightarrow+\infty$ the behavior of the multiplier $K(\rho)$ in the inequality

$$
K(\rho) \int_{\Pi_{\rho}}|u(y, z)|^{2} d y \leq\left\|u ; H^{2}(\Omega)\right\|^{2}, \quad u \in H^{2}(\Omega) .
$$

If $K(\rho)$ increases unboundedly as $\rho \rightarrow+\infty$ then, as above, Theorem 10.1.5 of [Birman and Solomyak 1980] ensures that the spectrum of the equation (4)-(7) stays discrete even in the case both sides of the peak are supplied with the tractionfree boundary conditions $(\mathrm{N})$ and, moreover, for any other boundary conditions from the list (5)-(7).

Proposition 11. Suppose that Assumption 6 is satisfied for $h=H$ and that

$$
\lim _{t \rightarrow \infty} \partial_{t}(\log H(t))=-\infty .
$$

Then for $\rho$ sufficiently large (19) holds true with

$$
K(\rho)=c \min \left\{H^{-4}(\rho), W_{H}(\rho), W_{H^{3}}(\rho)\right\} .
$$


Proof. It is sufficient to check the inequality (19) for smooth functions which vanish for $y<\rho$. We use the representation

$$
u(x)=u(y, z)=u_{0}(y)+z u_{1}(y)+u^{\perp}(y, z)
$$

where, for $y>\rho$, the component $u^{\perp}$ is subject to the orthogonality conditions

$$
\begin{aligned}
& \int_{\Upsilon(y)} u^{\perp}(y, z) d z=0, \\
& \int_{\Upsilon(y)} \partial_{z} u^{\perp}(y, z) d z=u^{\perp}(y, H(y))-u^{\perp}(y,-H(y))=0 .
\end{aligned}
$$

Let us process the integrals on the right-hand side of

$$
\int_{\Pi_{\rho}}\left|\nabla_{x}^{2} u(x)\right|^{2} d x=I_{1}+4 I_{2}+I_{3}
$$

where

$$
I_{1}:=\int_{\Pi_{\rho}}\left|\partial_{z}^{2} u(x)\right|^{2} d x, \quad I_{2}:=\int_{\Pi_{\rho}}\left|\partial_{y} \partial_{z} u(x)\right|^{2} d x, \quad I_{3}:=\int_{\Pi_{\rho}}\left|\partial_{y}^{2} u(x)\right|^{2} d x .
$$

Since $I_{1}=\int_{\rho}^{+\infty} \int_{\Upsilon(y)}\left|\partial_{z}^{2} u^{\perp}(y, z)\right|^{2} d z d y$ and since, by the orthogonality conditions in (21), inequality (13) holds here also, we find that

$$
I_{1} \geq c \int_{\Pi_{\rho}} H(y)^{-4}\left|u^{\perp}(x)\right|^{2} d x .
$$

For the last term in (22) we have

$$
I_{3}=\int_{\Pi_{\rho}}\left|\partial_{y}^{2} u_{0}(y)+z \partial_{y}^{2} u_{1}(y)+\partial_{y}^{2} u^{\perp}(y, z)\right|^{2} d x \geq J_{1}+J_{2}+2 J_{3}+2 J_{4},
$$

where

$$
\begin{array}{ll}
J_{1}=g \int_{\Pi_{\rho}}\left|\partial_{y}^{2} u_{0}(y)\right|^{2} d x, & J_{3}=g \int_{\Pi_{\rho}} \partial_{y}^{2} u_{0}(y) \partial_{y}^{2} u^{\perp}(y, z) d x \\
J_{2}=g \int_{\Pi_{\rho}}\left|z \partial_{y}^{2} u_{1}(y)\right|^{2} d x, & J_{4}=g \int_{\Pi_{\rho}} z \partial_{y}^{2} u_{1}(y) \partial_{y}^{2} u^{\perp}(y, z) d x
\end{array}
$$

We readily notice that according to the inequality (15) the estimates

$$
J_{1} \geq W_{H}(\rho) \int_{\rho}^{+\infty} 2 H(y)\left|u_{0}(y)\right|^{2} d y=W_{H}(\rho) \int_{\Pi_{\rho}}\left|u_{0}(y)\right|^{2} d x
$$


and

$$
\begin{aligned}
J_{2} & =\frac{2}{3} \int_{\rho}^{+\infty} H^{3}(y)\left|\partial_{y}^{2} u_{1}(y)\right|^{2} d y \geq \frac{2}{3} W_{H^{3}}(\rho) \int_{\rho}^{+\infty} H^{3}(y)\left|u_{1}(y)\right|^{2} d y \\
& =W_{H^{3}}(\rho) \int_{\Pi_{\rho}}\left|z u_{1}(y)\right|^{2} d x
\end{aligned}
$$

are fulfilled. For our purpose we need $W_{H}(\rho) \rightarrow+\infty$ and $W_{H^{3}}(\rho) \rightarrow+\infty$ for $\rho \rightarrow \infty$ and this we will assume.

Besides, by the Cauchy-Bunyakovsky-Schwarz inequality, we have

$$
\left|J_{3}\right| \leq J_{1}^{1 / 2}\left(\int_{\rho}^{+\infty} \frac{1}{2 H(y)}\left(\int_{\Upsilon(y)} \partial_{y}^{2} u^{\perp}(y, z) d z\right)^{2} d y\right)^{1 / 2} .
$$

We now deal with the inner integral in $z$ in the last expression. To this end, we take into account the orthogonality conditions (21) and the trace inequality. We differentiate the first equality in (21) twice with respect to $y$ and obtain

$$
\begin{array}{r}
\sum_{ \pm}\left(2 \partial_{y} u^{\perp}(y, \pm H(y)) \partial_{y} H(y)+u^{\perp}(y, \pm H(y)) \partial_{y}^{2} H(y) \pm \partial_{z} u^{\perp}(y, \pm H(y))\left(\partial_{y} H(y)\right)^{2}\right) \\
+\int_{\Upsilon(y)} \partial_{y}^{2} u^{\perp}(y, z) d z=0 .
\end{array}
$$

Thus,

$$
\begin{aligned}
& \left(\int_{\Upsilon(y)} \partial_{y}^{2} u^{\perp}(y, z) d z\right)^{2} \\
& \quad \leq c \sum_{ \pm}\left(\left|\partial_{z} u^{\perp}(y, \pm H(y))\right|^{2}\left|\partial_{y} H(y)\right|^{4}+\left|u^{\perp}(y, \pm H(y))\right|^{2}\left|\partial_{y}^{2} H(y)\right|^{2}\right. \\
& \left.+\left|\partial_{y} u^{\perp}(y, \pm H(y))\right|^{2}\left|\partial_{y} H(y)\right|^{2}\right) .
\end{aligned}
$$

For the first two terms between the brackets we use the trace inequality

$$
\left|\partial_{z} u^{\perp}(y, \pm H(y))\right|^{2}|H(y)|^{2}+\left|u^{\perp}(y, \pm H(y))\right|^{2} \leq c|H(y)|^{3} \int_{\Upsilon(y)}\left|\partial_{z}^{2} u^{\perp}(y, z)\right|^{2} d z .
$$

For the third term, we write down the chain of inequalities

$$
\begin{aligned}
& \left|\partial_{y} u^{\perp}(y, \pm H(y))\right|^{2} \\
& \leq c H(y) \int_{\Upsilon(y)}\left|\partial_{y} \partial_{z} u^{\perp}(y, z)\right|^{2} d z+c|H(y)|^{-2}\left(\int_{\Upsilon(y)} \partial_{y} u^{\perp}(y, z) d z\right)^{2} \\
& \leq c H(y) \int_{\Upsilon(y)}\left|\partial_{y} \partial_{z} u^{\perp}(y, z)\right|^{2} d z \\
& \quad+2 c\left(\frac{\partial_{y} H(y)}{H(y)}\right)^{2}\left(\left|u^{\perp}(y, H(y))\right|^{2}+\left|u^{\perp}(y,-H(y))\right|^{2}\right) .
\end{aligned}
$$


As a result, we find that

$$
\begin{array}{r}
\left|\int_{\Upsilon(y)} \partial_{y}^{2} u^{\perp}(y, z) d z\right|^{2} \leq c\left(\left|\partial_{y} H(y)\right|^{4} H(y)+\left|\partial_{y}^{2} H(y)\right|^{2}|H(y)|^{3}\right) \int_{\Upsilon(y)}\left|\partial_{z}^{2} u^{\perp}(y, z)\right|^{2} d z \\
+c\left|\partial_{y} H(y)\right|^{2}|H(y)| \int_{\Upsilon(y)}\left|\partial_{y} \partial_{z} u^{\perp}(y, z)\right|^{2} d z .
\end{array}
$$

The final inequality for the integral $J_{3}$ takes the form

$$
\left|J_{3}\right| \leq c_{1}(\rho) J_{1}^{1 / 2} I_{1}^{1 / 2}+c_{2}(\rho) J_{1}^{1 / 2} K_{1}^{1 / 2}
$$

where $K_{1}=\left\|\partial_{y z}^{2} u^{\perp} ; L_{2}\left(\Pi_{\rho}\right)\right\|^{2}$ and

$$
\begin{aligned}
& c_{1}(\rho)=c \sup _{y \in[\rho,+\infty)}\left(\left|\partial_{y} H(y)\right|^{2}+\left|\partial_{y}^{2} H(y)\right||H(y)|\right), \\
& c_{2}(\rho)=c \sup _{y \in[\rho,+\infty)}\left|\partial_{y} H(y)\right| .
\end{aligned}
$$

Both suprema tend to 0 for $\rho \rightarrow+\infty$. A similar argument shows that

$$
\left|J_{4}\right| \leq c_{1}(\rho) J_{2}^{1 / 2} I_{1}^{1 / 2}+c_{2}(\rho) J_{2}^{1 / 2} K_{1}^{1 / 2} .
$$

It remains to process the second term in (22), that is,

$$
\begin{aligned}
& I_{2}=\int_{\Pi_{\rho}}\left|z \partial_{y} u_{1}(y)+\partial_{y} \partial_{z} u^{\perp}(y, z)\right|^{2} d x \\
& =\int_{\Pi_{\rho}}\left|\partial_{y} \partial_{z} u^{\perp}(y, z)\right|^{2} d x+\int_{\Pi_{\rho}}\left|\partial_{y} u_{1}(y)\right|^{2} d x+2 \int_{\Pi_{\rho}} \partial_{y} u_{1}(y) \partial_{y} \partial_{z} u^{\perp}(y, z) d x . \\
& \text { " } \\
& K_{1} \\
& \bigwedge K_{2} \\
& 2 K_{3}
\end{aligned}
$$

So it follows that

$$
K_{1}=I_{2}-K_{2}-2 K_{3} \leq I_{2}+2\left|K_{3}\right| .
$$

We continue by estimating the integral $K_{3}$ :

$$
\begin{aligned}
& \left|K_{3}\right|=\left|\int_{\rho}^{+\infty} \partial_{y} u_{1}(y) \int_{\Upsilon(y)} \partial_{y} \partial_{z} u^{\perp}(y, z) d z d y\right| \\
& \leq\left(\int_{\rho}^{+\infty} G_{H^{3}, \rho}(y)\left|\partial_{y} u_{1}(y)\right|^{2} d y\right)^{\frac{1}{2}}\left(\int_{\rho}^{+\infty} G_{H^{3}, \rho}(y)^{-1}\left|\int_{\Upsilon(y)} \partial_{y} \partial_{z} u^{\perp}(y, z) d z\right|^{2} d y\right)^{\frac{1}{2}} \\
& \leq c J_{2}^{1 / 2}\left(\int_{\rho}^{+\infty} G_{H^{3}, \rho}(y)^{-1}\left|\int_{\Upsilon(y)} \partial_{y} \partial_{z} u^{\perp}(y, z) d z\right|^{2} d y\right)^{1 / 2} .
\end{aligned}
$$


Differentiating the second formula (21) with respect to $y$ yields

By the trace inequality we find that

$$
\int_{\Upsilon(y)} \partial_{y} \partial_{z} u^{\perp}(y, z) d z+\sum_{ \pm} \partial_{z} u^{\perp}(y, \pm H(y)) \partial_{y} H(y)=0 .
$$

$$
\begin{aligned}
\left|\int_{\Upsilon(y)} \partial_{y} \partial_{z} u^{\perp}(y, z) d z\right|^{2} & =\left(\sum_{ \pm} \partial_{z} u^{\perp}(y, \pm H(y))\right)^{2}\left|\partial_{y} H(y)\right|^{2} \\
& \leq c\left|H(y) \| \partial_{y} H(y)\right|^{2} \int_{\Upsilon(y)}\left|\partial_{z}^{2} u^{\perp}(y, z)\right|^{2} d z .
\end{aligned}
$$

Thus, from the relation (18), which implies (17), we get

$$
\left|K_{3}\right| \leq c \sup _{y \in[\rho,+\infty)}\left\{\left|G_{H^{3}, \rho}(y)\right|^{-1 / 2}\left|\partial_{y} H(y)\right||H(y)|^{1 / 2}\right\} J_{2}^{1 / 2} I_{1}^{1 / 2} \leq c J_{2}^{1 / 2} I_{1}^{1 / 2} .
$$

We find by combining (29) and (30) that

$$
K_{1} \leq I_{2}+c J_{2}^{1 / 2} I_{1}^{1 / 2}
$$

and so (27) and (28) yield, respectively,

$$
\begin{aligned}
& \left|J_{3}\right| \leq c_{1}(\rho) J_{1}^{1 / 2} I_{1}^{1 / 2}+c_{2}(\rho) J_{1}^{1 / 2}\left(I_{2}+c J_{2}^{1 / 2} I_{1}^{1 / 2}\right)^{1 / 2}, \\
& \left|J_{4}\right| \leq c_{1}(\rho) J_{2}^{1 / 2} I_{1}^{1 / 2}+c_{2}(\rho) J_{2}^{1 / 2}\left(I_{2}+c J_{2}^{1 / 2} I_{1}^{1 / 2}\right)^{1 / 2} .
\end{aligned}
$$

Using first (22) and (24), next (31) and (32) for $\rho$ large enough, and finally (23), (25) and (26) we conclude that indeed

$$
\begin{aligned}
\left\|\nabla_{x}^{2} u ; L_{2}\left(\Pi_{\rho}\right)\right\|^{2} & =I_{1}+4 I_{2}+I_{3} \\
& \geq I_{1}+4 I_{2}+J_{1}+J_{2}+2 J_{3}+2 J_{4} \\
& \geq \frac{1}{2}\left(I_{1}+4 I_{2}+J_{1}+J_{2}\right) \geq \frac{1}{2}\left(I_{1}+J_{1}+J_{2}\right) \\
& \geq c \min \left\{H^{-4}(\rho), W_{H}(\rho), W_{H^{3}}(\rho)\right\}\left\|u ; L_{2}\left(\Pi_{\rho}\right)\right\|^{2},
\end{aligned}
$$

whenever $\rho$ is large enough.

\section{Traction-free boundaries: $N-N$}

Theorem 12. Suppose that $H$ satisfies Assumption 6 with $h=H$ and that

$$
\lim _{t \rightarrow \infty} \partial_{t}(\log H(t))=-\infty .
$$

Then the embedding $H^{2}(\Omega) \hookrightarrow L_{2}(\Omega)$ is compact and the spectrum of the problem (4) with the Neumann boundary conditions (6) on both sides of the peak is discrete. 
Proof. By Proposition 11, $K(\rho)$ can be estimated as in (20). Assumption 6 implies that Lemma 10 holds true and hence $K(\rho) \rightarrow+\infty$ for $\rho \rightarrow+\infty$. One concludes as in the proof of Theorem 5 through an approximation by compact operators.

Remark 12.1. Note that the functions $H(y)=y^{-\alpha}$ and $H(y)=\exp (-\alpha y), \alpha>0$, do not satisfy the requirement in (2) or (33). The functions $H(y)=\exp \left(-y^{1+\alpha}\right)$ with $\alpha>0$ however do.

\section{An incomplete Dirichlet condition: $M-N$}

In this section the boundary conditions only contain a single stable condition

$$
u(x)=0, \quad x \in \Sigma_{\rho}^{+}\left(\text {or } x \in \Sigma_{\rho}^{-}\right) .
$$

Theorem 13. Suppose that $\lim _{y \rightarrow \infty} H(y)^{-3} G_{H}(y)=+\infty$. Then the problem in (4) with the boundary condition as in (34) has discrete spectrum.

Remark 13.1. The functions $H(y)=y^{-1-\alpha}$ with $\alpha>0$ meet the condition in Theorem 13.

Proof. By (34), Friedrich's inequality holds on the section $\Upsilon(y)$ and, consequently,

$$
\left\|\mathscr{A} \partial_{z} u ; L_{2}\left(\Pi_{\rho}\right)\right\|^{2} \geq c\left\|\mathscr{A} H^{-2} u ; L_{2}\left(\Pi_{\rho}\right)\right\|^{2}
$$

for every positive weight function $y \mapsto \mathscr{A}(y)$. The function $v=\partial_{z} u$ can be represented as the sum $v(x)=v_{0}(y)+v^{\perp}(x)$ where, for $y>\rho$, the component $v^{\perp}$ satisfies the first condition in (21). Therefore,

$$
\begin{aligned}
& \int_{\Pi_{\rho}}\left|\nabla_{x}^{2} u(x)\right|^{2} d x \\
& \geq \int_{\Pi_{\rho}}\left|\nabla_{x} v(x)\right|^{2} d x \\
& \geq \int_{\rho}^{+\infty} 2 H(y)\left|\partial_{y} v_{0}(y)\right|^{2} d y+\int_{\Pi_{\rho}}\left|\partial_{z} v^{\perp}(y, z)\right|^{2} d x+2 \int_{\Pi_{\rho}} \partial_{y} v_{0}(y) \partial_{y} v^{\perp}(y, z) d x \\
& =: I_{4}+I_{5}+2 I_{6} .
\end{aligned}
$$

Setting $Z_{H}(y)=H(y)^{-1} G_{H}(y)$, we get

$$
\begin{aligned}
I_{4} \geq \int_{\rho}^{+\infty} 2 G_{H}(y)\left|v_{0}(y)\right|^{2} d y & \geq \int_{\rho}^{+\infty} 2 Z_{H}(y) H(y)\left|v_{0}(y)\right|^{2} d y \\
& =\left\|Z_{H} v_{0} ; L_{2}\left(\Pi_{\rho}\right)\right\|^{2} .
\end{aligned}
$$

Friedrich's inequality implies

$$
I_{5}=\left\|\partial_{z} v^{\perp} ; L_{2}\left(\Pi_{\rho}\right)\right\| \geq c\left\|H^{-2} v^{\perp} ; L_{2}\left(\Pi_{\rho}\right)\right\|^{2} .
$$


Furthermore,

$$
\begin{aligned}
I_{6} & =\int_{\Pi_{\rho}} \partial_{y} v_{0}(y) \partial_{y} v^{\perp}(y, z) d x \leq I_{5}^{1 / 2}\left(\int_{\rho}^{+\infty} \frac{1}{2 H(y)}\left|\int_{\Upsilon(y)} \partial_{y} v^{\perp}(y, z) d z\right|^{2}\right)^{1 / 2} \\
& \leq I_{5}^{1 / 2}\left(\int_{\rho}^{+\infty} \frac{1}{2 H(y)}\left|v^{\perp}(y, H(y)) \partial_{y} H(y)-v^{\perp}(y,-H(y)) \partial_{y} H(y)\right|^{2}\right)^{1 / 2} .
\end{aligned}
$$

Thus $I_{6} \leq c\left|\partial_{y} H(\rho)\right| I_{5}^{1 / 2} I_{6}^{1 / 2}$ holds and hence

$$
\left\|\nabla_{x}^{2} u ; L_{2}\left(\Pi_{\rho}\right)\right\|^{2} \geq c\left\|\min \left\{H^{-4} ; H^{-3} G_{H}\right\} u ; L_{2}\left(\Pi_{\rho}\right)\right\|^{2} .
$$

Compactness and hence the discrete spectrum follow from the assumption on $H$.

\section{References}

[Adams and Fournier 2003] R. A. Adams and J. J. F. Fournier, Sobolev spaces, 2nd ed., Pure and Applied Mathematics 140, Elsevier/Academic Press, Amsterdam, 2003.

[Bakharev and Nazarov 2009] F. L. Bakharev and S. A. Nazarov, "On the structure of the spectrum of a problem in the theory of elasticity for a body with a supersharp peak", Sibirsk. Mat. Zh. 50:4 (2009), 746-756. In Russian; translated in Sib. Math. J. 50:4 (2009), 587-595.

[van den Berg 1984] M. van den Berg, "On the spectrum of the Dirichlet Laplacian for horn-shaped regions in $\mathbf{R}^{n}$ with infinite volume”, J. Funct. Anal. 58:2 (1984), 150-156.

[van den Berg and Lianantonakis 2001] M. van den Berg and M. Lianantonakis, "Asymptotics for the spectrum of the Dirichlet Laplacian on horn-shaped regions", Indiana Univ. Math. J. 50:1 (2001), 299-333.

[Birman and Solomyak 1980] M. S. Birman and M. Z. Solomyak, Спектралная теория самосопряженных операторов в гилбертовом пространстве, Leningrad Univ., 1980. Translated as Spectral theory of selfadjoint operators in Hilbert space, Reidel, Dordrecht, 1987.

[Boyarchenko and Levendorskii 2000] S. I. Boyarchenko and S. Z. Levendorskii, "Spectral asymptotics of the Neumann Laplacian on oscillating horns", Russ. J. Math. Phys. 7:1 (2000), 1-14.

[Courant and Hilbert 1953] R. Courant and D. Hilbert, Methods of mathematical physics, vol. 1, Interscience, New York, 1953.

[Davies and Simon 1992] E. B. Davies and B. Simon, "Spectral properties of Neumann Laplacian of horns", Geom. Funct. Anal. 2:1 (1992), 105-117.

[Evans and Harris 1987] W. D. Evans and D. J. Harris, "Sobolev embeddings for generalized ridged domains", Proc. London Math. Soc. (3) 54:1 (1987), 141-175.

[Gazzola et al. 2010] F. Gazzola, H.-C. Grunau, and G. Sweers, Polyharmonic boundary value problems: Positivity preserving and nonlinear higher order elliptic equations in bounded domains, Lecture Notes in Mathematics 1991, Springer, Berlin, 2010.

[Hempel et al. 1991] R. Hempel, L. A. Seco, and B. Simon, "The essential spectrum of Neumann Laplacians on some bounded singular domains", J. Funct. Anal. 102:2 (1991), 448-483.

[Ivrii 1999] V. Ivrii, "Precise spectral asymptotics for Neumann Laplacian in domains with cusps", Appl. Anal. 71:1-4 (1999), 139-147.

[Jakšić 1993] V. Jakšić, "On the spectrum of the Neumann Laplacian of long-range horns: a note on the Davies-Simon theorem”, Proc. Amer. Math. Soc. 119:2 (1993), 663-669. 
[Jakšić et al. 1992] V. Jakšić, S. Molčanov, and B. Simon, "Eigenvalue asymptotics of the Neumann Laplacian of regions and manifolds with cusps", J. Funct. Anal. 106:1 (1992), 59-79.

[Kovařík 2011] H. Kovařík, "Eigenvalue asymptotic of Robin Laplace operators on two-dimensional domains with cusps", J. Lond. Math. Soc. (2) 83:1 (2011), 256-271.

[Krylov and Tilman 2004] V. V. Krylov and F. J. B. S. Tilman, "Acoustic 'black holes' for flexural waves as effective vibration dampers", J. Sound Vib. 274 (2004), 605-619.

[Maz'ya and Poborchi 1997] V. G. Maz'ya and S. V. Poborchi, Differentiable functions on bad domains, World Scientific, River Edge, NJ, 1997.

[Maz'ya and Poborchii 2006] V. G. Maz'ya and S. V. Poborchii, “Theorems for embedding Sobolev spaces on domains with a peak and on Hölder domains", Algebra i Analiz 18:4 (2006), 95-126.

[Mikhlin 1970] S. G. Mikhlin, Вариационные методы в математической физике, 2nd ed., Nauka, Moscow, 1970. First edition translated as Variational methods in mathematical physics, Pergamon Press, Oxford, 1964.

[Mironov 1988] M. A. Mironov, "Propagation of a flexural wave in a plate whose thickness decreases smoothly to zero in a finite interval”, Soviet Phys. Acoust. 34 (1988), 318-319.

[Nazarov 1999] S. A. Nazarov, "Polynomial property of selfadjoint elliptic boundary value problems, and the algebraic description of their attributes", Uspekhi Mat. Nauk 54:5(329) (1999), 77142. In Russian; translated in Russian Math. Surveys 54:5 (1999), 947-1014.

[Nazarov 2002] S. A. Nazarov, Asymptotic theory of thin plates and rods, vol. 1: Dimension reduction and integral estimates, Nauchnaya Kniga, Novosibirsk, 2002.

[Nazarov 2008] S. A. Nazarov, "On the spectrum of a problem in the theory of elasticity for a spiked body”, Sibirsk. Mat. Zh. 49:5 (2008), 1105-1127.

[Nazarov 2009] S. A. Nazarov, "On the essential spectrum of boundary value problems for systems of differential equations in a bounded domain with a cusp", Funktsional. Anal. i Prilozhen. 43:1 (2009), 55-67. In Russian; translated in Funct. Anal. Appl. 43:1 (2009), 44-54.

[Nazarov and Taskinen 2008] S. A. Nazarov and J. Taskinen, "On the spectrum of the Steklov problem in a domain with a peak", Vestnik Sankt-Pet. Univ. Ser. 1 Mat. Mekh. Astr. 2008:1 (2008), 56-65. In Russian; translated in Vestnik St. Petersburg Univ. Math. 41:1 (2008), 45-52.

[Rellich 1948] F. Rellich, "Das Eigenwertproblem von $\Delta u+\lambda u=0$ in Halbröhren", pp. 329-344 in Studies and essays presented to R. Courant on his 60th birthday, Interscience, New York, 1948.

[Rozenbljum 1972] G. V. Rozenbljum, "The eigenvalues of the first boundary value problem in unbounded domains", Mat. Sb. (N.S.) 89:(131) (1972), 234-247. In Russian; translated in Math. USSR Sb. 18 (1972), 235-248 (1973).

[Simon 1992] B. Simon, “The Neumann Laplacian of a jelly roll”, Proc. Amer. Math. Soc. 114:3 (1992), 783-785.

Received 7 Mar 2012. Revised 21 Jul 2012. Accepted 28 Aug 2012.

FEDOR L. BAKHAREV: fbakharev@yandex.ru

Mathematics and Mechanics Faculty, St. Petersburg State University, 198904 St. Petersburg, Russia

SERGEY A. NAZARov: srgnazarov@yahoo.co.uk

Institute of Problems of Mechanical Engineering, Russian Academy of Sciences,

199178 St. Petersburg, Russia

Current address: Mathematics and Mechanics Faculty, St. Petersburg State University,

Universitetsky pr., 28, 198504, Stary Peterhof, Russia

GUIDO H. SWEERS: gsweers@math.uni-koeln.de

Mathematisches Institut, Universität zu Köln, D-50931 Cologne, Germany

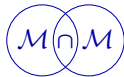




\section{EDITORIAL BOARD}

ANTONIO CARCATERRA ERIC A. CARLEN

FRANCESCO DELL'ISOLA

RAFFAELE ESPOSITO

ALBERT FANNJIANG

Gilles A. FrancFort

PiERANGElo MARCATI

JEAN-JACQUES MARIGO

PETER A. MARKOWICH MARTIN OSTOJA-STARZEWSKI

PIERRE SEPPECHER

DAVID J. STEIGMANN

PAUl STEINMANN

PierRe M. Suquet

\section{MANAGING EDITORS}

MICOL AMAR

CORRADO LATTANZIO

ANGELA MADEO

MARTIN OSTOJA-STARZEWSKI

\section{ADVISORY BOARD}

ADNAN AKAY

Holm AltenbaCH

MICOL AMAR

HARM ASKES

TEODOR ATANACKOVIĆ

VICTOR BERDICHEVSKY

GuY BouchitTÉ

ANDREA BRAIDES

ROBERTO CAMASSA

MAURO CARFORE

ERIC DARVE

FELIX DARVE

ANNA DE MASI

Gianpietro Del Piero

EMMANUELE Di BENEDETTO

BERNOLD FIEDLER

IRENE M. GAMBA

SERGEY GAVRILYUK

TIMOTHY J. HEALEY

DOMINIQUE JEULIN

ROGER E. KHAYAT

CORRADO LATTANZIO

ROBERT P. LIPTON

ANGELO LUONGO

ANGEla MadeO

JUAN J. MANFREDI

CARLO MARCHIORO

GÉrard A. MAUGin

ROBERTO NATALINI

PATRIZIO NEFF

ANDREY PIATNITSKI

ERrico Presutti

MARIO PUlVIRENTI

LuCiO RuSSO

Miguel A. F. SANJUAN

Patrick SElvadurai

ALEXANDER P. SEYRANIAN

MIROSLAV ŠILHAVÝ

GUIDO SWEERS

ANTOINETTE TORDESILLAS

LEV TRUSKINOVSKY

JUAN J. L. VELÁZQUEZ

VINCENZO VESPRI

ANGELO VULPIANI msp.org/memocs

Università di Roma “La Sapienza”, Italia

Rutgers University, USA

(CO-CHAIR) Università di Roma "La Sapienza", Italia

(TREASURER) Università dell'Aquila, Italia

University of California at Davis, USA

(CO-CHAIR) Université Paris-Nord, France

Università dell' Aquila, Italy

École Polytechnique, France

DAMTP Cambridge, UK, and University of Vienna, Austria

(CHAIR MANAGING EDITOR) Univ. of Illinois at Urbana-Champaign, USA

Université du Sud Toulon-Var, France

University of California at Berkeley, USA

Universität Erlangen-Nürnberg, Germany

LMA CNRS Marseille, France

Università di Roma “La Sapienza”, Italia

Università dell' Aquila, Italy

Université de Lyon-INSA (Institut National des Sciences Appliquées), France (CHAIR MANAGING EDITOR) Univ. of Illinois at Urbana-Champaign, USA

Carnegie Mellon University, USA, and Bilkent University, Turkey

Otto-von-Guericke-Universität Magdeburg, Germany

Università di Roma "La Sapienza”, Italia

University of Sheffield, UK

University of Novi Sad, Serbia

Wayne State University, USA

Université du Sud Toulon-Var, France

Università di Roma Tor Vergata, Italia

University of North Carolina at Chapel Hill, USA

Università di Pavia, Italia

Stanford University, USA

Institut Polytechnique de Grenoble, France

Università dell'Aquila, Italia

Università di Ferrara and International Research Center MEMOCS, Italia

Vanderbilt University, USA

Freie Universität Berlin, Germany

University of Texas at Austin, USA

Université Aix-Marseille, France

Cornell University, USA

École des Mines, France

University of Western Ontario, Canada

Università dell' Aquila, Italy

Louisiana State University, USA

Università dell' Aquila, Italia

Université de Lyon-INSA (Institut National des Sciences Appliquées), France

University of Pittsburgh, USA

Università di Roma "La Sapienza", Italia

Université Paris VI, France

Istituto per le Applicazioni del Calcolo "M. Picone", Italy

Universität Duisburg-Essen, Germany

Narvik University College, Norway, Russia

Università di Roma Tor Vergata, Italy

Università di Roma "La Sapienza", Italia

Università di Roma “Tor Vergata", Italia

Universidad Rey Juan Carlos, Madrid, Spain

McGill University, Canada

Moscow State Lomonosov University, Russia

Academy of Sciences of the Czech Republic

Universität zu Köln, Germany

University of Melbourne, Australia

École Polytechnique, France

Bonn University, Germany

Università di Firenze, Italia

Università di Roma La Sapienza, Italia

MEMOCS (ISSN 2325-3444 electronic, 2326-7186 printed) is a journal of the International Research Center for the Mathematics and Mechanics of Complex Systems at the Università dell'Aquila, Italy.

Cover image: "Tangle" by @ John Horigan; produced using the Context Free program (contextfreeart.org).

\section{PUBLISHED BY}

mathematical sciences publishers

nonprofit scientific publishing

http://msp.org/

(C) 2013 Mathematical Sciences Publishers 
Mathematics and Mechanics of Complex Systems vol. 1 no. 2

Self-organized stochastic tipping in slow-fast dynamical systems

Mathias Linkerhand and Claudius Gros

Well-posedness for dislocation-based gradient

viscoplasticity, II: general nonassociative monotone plastic flows

Sergiy Nesenenko and Patrizio Neff

Symmetry classes for even-order tensors

Marc Olive and Nicolas Auffray

A direct approach to nonlinear shells with application to surface-substrate interactions

Miroslav Šilhavý

A sufficient condition for a discrete spectrum of the Kirchhoff plate with an infinite peak

Fedor L. Bakharev, Sergey A. Nazarov and Guido H. Sweers

MEMOCS is a journal of the International Research Center for the Mathematics and Mechanics of Complex Systems at the Università dell' Aquila, Italy.

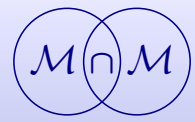

